\title{
INVESTIGACIONES
}

\section{Elaboración y validación psicométrica del cuestionario de convivencia escolar para la no violencia (CENVI)}

\author{
Development and psychometric validation of the questionnaire \\ of school life for nonviolence (CENVI)
}

\author{
Flavio Muñoz Troncoso ${ }^{a}$, Sandra Becerra Peña ${ }^{b}$, Enrique Riquelme $^{c}$ \\ a Universidad La República, sede Temuco. Correo electrónico: flaviomunoz@gmail.com \\ ${ }^{\text {b}}$ Facultad de Educación, Universidad Católica de Temuco. Correo electrónico: sbecerra@uct.cl \\ c Universidad Católica de Temuco. Correo electrónico: eriquelme@uct.cl
}

\begin{abstract}
RESUMEN
El estudio presenta el proceso de elaboración y validación psicométrica del cuestionario de convivencia escolar para la no violencia. El problema surge de la carencia de instrumentos que evalúen de manera conjunta y desde la perspectiva de los estudiantes, los tipos de violencia manifestados en la escuela y la gestión de la convivencia escolar. Las dimensiones del instrumento se elaboraron en función de los diagnósticos nacionales de violencia escolar, los estándares de desempeño entregados por el Ministerio de Educación (MINEDUC) y tomando orientaciones de cuestionarios existentes respecto de violencia y convivencia en el medio escolar. Participaron 1410 estudiantes de $5^{\circ}$ a $8^{\circ}$ año básico de establecimientos educacionales de Temuco, Chile. Se aplicó análisis factorial confirmatorio (AFC) y análisis de confiabilidad interna. Los análisis reportan confiabilidad interna del instrumento y un ajuste adecuado al modelo. El artículo finaliza discutiendo las proyecciones y limitaciones del instrumento y proceso de validación.
\end{abstract}

Palabras clave: prevención de violencia, convivencia, gestión escolar, análisis factorial confirmatorio

\begin{abstract}
This paper presents the process of development and psychometric validation of the questionnaire: school life for nonviolence (CENVI). The problem arises from the lack of tools to assess both the types of violence and management of school life from the perspective of students. The instrument dimensions were developed based on national assessments of school violence and standards provided by the Ministry of Education, and the orientations of existing questionnaires regarding violence and coexistence in schools. 1410 students from fifth to 8th grade of educational establishments of Temuco, Chile participated in the study. Confirmatory factor analysis (CFA) and internal reliability analysis were applied. Analyses reported internal reliability of the instrument and an appropriate adjustment of the model. The article concludes by discussing the projections and limitations of the instrument and validation process.
\end{abstract}

Key words: violence prevention, coexistence, school management, confirmatory factor analysis 


\section{INTRODUCCIÓN}

La institución educativa es la encargada de promover una convivencia armónica entre los estudiantes, abriendo espacios para aprender el respeto, la tolerancia, la solidaridad y la inclusión (MINEDUC, 2014). El desafío para toda escuela es que su quehacer sea valorado como instancias de formación para aprender a convivir fortaleciendo el buen trato y la no violencia. Las normas de convivencia escolar presentes en los manuales y reglamentos aportan a este desafío. Todo lo anterior subyace en una apropiada gestión de la convivencia, que influirá sobre la interacción de los estudiantes y sus resultados de aprendizajes, lo que trascenderá del espacio educativo a lo largo de sus vidas.

La violencia escolar presenta diversas formas de manifestación descritas como física, verbal, exclusión social, disrupción en el aula y a través de medios tecnológicos (Dobarro, Álvarez-García y Núñez, 2014; Álvarez-García, Núñez y Dobarro, 2013). Se vincula de manera perjudicial con los resultados de aprendizaje (Murillo, 2011), y a efectos psicosociales negativos (Cava, Buelga, Musitu y Murgui, 2010; Albaladejo, 2011; Sánchez y Cerezo, 2011). Además, los estudiantes que son víctima de violencia pueden presentar diversos efectos psicológicos inmediatos, los que podrían prolongarse dependiendo del tipo de violencia que sufren (Berger y Lisboa, 2009; Hinduja y Patchin, 2010; Guerra et al., 2011; López, Bilbao y Rodríguez, 2012; Buelga, Cava y Musitu, 2012).

La aproximación conceptual de la convivencia que asume la política pública, se fundamenta en dos paradigmas, uno de ellos refiere al control y la sanción y el otro a la creación de condiciones para establecer una convivencia democrática, respetando los derechos de cada integrante de la comunidad educativa (Magendzo, Toledo y Gutiérrez, 2013). En esta lógica se busca el aseguramiento de la sana convivencia por medio de leyes y normativas. Por ejemplo, el Art. $2^{\circ}$ de la Ley General de Educación (LGE) está centrado en la formación integral, que supera el aprendizaje de contenidos curriculares e incorpora la convivencia con el medio (Ley $N^{\circ}$ 20370, 2009); El Artículo $10^{\circ}$ refiere a los derechos y deberes de la comunidad educativa, centrados en la dignidad, respeto, tolerancia y la no discriminación, y el Artículo $15^{\circ}$ profundiza en la participación de los integrantes de la comunidad educativa a través de sus diversos estamentos.

Lo señalado se enlaza con los estándares indicativos de desempeño elaborados por MINEDUC en 2014, con la finalidad que la comunidad educativa evalúe sus prácticas de gestión. Dichos estándares en su dimensión de formación y convivencia establecen pautas para la formación, convivencia y participación y vida democrática (MINEDUC, 2014). De esta manera, se resume que la normativa vigente respecto a convivencia escolar se centra en un enfoque formativo para el desarrollo de todas las dimensiones del ser humano (MINEDUC, 2015), lo que influiría en la prevención de situaciones de riesgo de violencia. La responsabilidad queda remitida a la comunidad educativa, que asume el rol de garante para asegurar la sana convivencia, propiciando ambientes para el desarrollo integral de los estudiantes y cumplimiento de normas que resguardan sus deberes y derechos, según lo establece la Ley Sobre Violencia Escolar (Ley N² 20536, 2011).

1.1 SOBRE LAS FORMAS DE EXPLORACIÓN DE LA VIOLENCIA ESCOLAR: INSTRUMENTOS EXISTENTES

El interés por investigar la violencia en los centros educativos ha generado instrumentos con diversas características. Por ejemplo, el instrumento de auto-reporte adaptado en 
Chile denominado "Cuestionario de Secundaria de Maltrato entre Iguales por abuso de Poder" (MIAP), adaptado y validado para Chile inicialmente por Lecannelier et al. (2011). Según Dan Olweus, citado en Marín-Martínez y Reidl (2013), de los instrumentos de autoreporte, el más utilizado es el Bully/Victim Questionnaire (BVQ) cuya consistencia interna es satisfactoria, con valores de fiabilidad superiores a .80 .

Se ha destacado el instrumento Español de Álvarez, Álvarez, Gonzáles, Núñez y González-Pienda (2006) "Cuestionario de Violencia Escolar" (CUVE), adaptado a Chile por Castro y Vargas en el año 2009 (citado en Guerra, Castro y Vargas, 2011) con posteriores validaciones respecto a sus propiedades psicométricas llevadas a cabo por Álvarez-García, Núñez, Rodríguez, Álvarez, y Dobarro (2011) y Álvarez et al (2013). Siendo además utilizado en un estudio comparativo de violencia escolar entre Chile y España (Guerra et al., 2011).

En el ámbito de la convivencia son escasos los instrumentos que exploren elementos de gestión. En México se elaboró un Cuestionario de Convivencia Escolar que cuenta con 183 ítems que exploran las dimensiones de inclusión, democracia y paz (Chaparro, Caso, Fierro y Díaz, 2015; Chaparro, Caso, Díaz y Urias, 2012). La premisa que subyace a este cuestionario es que la política y la cultura institucional en contextos de gestión escolar, incide en las dinámicas que se visualizan de la convivencia en los distintos espacios del establecimiento educacional.

En resumen, en Chile se han replicado varios estudios de carácter psicométrico en procesos de adaptación y validación, que han reportado datos de la prevalencia de la violencia escolar en el país (Instituto de Evaluación y asesoramiento Educativo, 2005; Guerra, Castro y Vargas, 2011; Lecannelier et al., 2011; López, Bilbao y Rodriguez, 2012). Además se ha explorado la violencia escolar desde distintos enfoques, por ejemplo, estudios sobre drogas en población escolar (Servicio Nacional para la Prevención y Rehabilitación del Consumo de Drogas y Alcohol (2006), de convivencia en las escuelas MINEDUC (2015) y el estudio de violencia en el ámbito escolar (MINEDUC y Universidad Alberto Hurtado, 2006).

De esta manera los últimos años de investigación sobre violencia escolar, han permitido el desarrollo de diversos instrumentos para medir sus formas de manifestación y prevalencia en la escuela. Además, se ha determinado que la convivencia escolar tiene un rol preponderante como elemento facilitador de los aprendizajes (Cid, 2004; Murillo, 2011), junto con posibilitar la prevención de la violencia (Chaparro et al., 2012), que se daría mediante un adecuado manejo por parte de la institución educativa.

Por todo expuesto, se considera que a la fecha no existen instrumentos que evalúen de manera conjunta y desde la percepción de los estudiantes, los tipos de violencia manifiestos en la escuela y la gestión de la convivencia escolar. En este contexto, se ha propuesto como objetivo el diseñar y validar un instrumento con dichas características, que permita diagnosticar la realidad de ambas dimensiones en establecimientos educacionales de las diferentes dependencias administrativas del sistema escolar chileno.

\section{MÉTODO}

\subsection{PARTICIPANTES}

Participaron 1410 estudiantes, cuyas edades oscilaron entre 10 y 14 años $(M=12,4$; DT=0,9). 729 fueron hombres y 681 mujeres, quienes cursaban desde $5^{\circ}$ a $8^{\circ}$ año de enseñanza general básica en seis establecimientos educacionales urbanos de la ciudad de Temuco, Chile (Ver tabla 1). De ellos, 431 estudiantes pertenecen a establecimientos de 
dependencia administrativa municipal, 564 del sistema particular subvencionado y 415 del sistema particular pagado. Se cauteló la participación voluntaria de los participantes en la investigación, que se realizó como una actividad curricular, asociada a la asignatura de orientación o consejo de curso. Lo anterior autorizado y coordinado por el director de cada establecimiento educativo.

Tabla 1: Frecuencia de características demográficas de los participantes

\begin{tabular}{|c|c|c|c|}
\hline & & Frecuencia & Porcentaje \\
\hline \multirow[t]{2}{*}{ Género } & Hombre & 729 & 51,7 \\
\hline & Mujer & 681 & 48,3 \\
\hline \multirow[t]{2}{*}{ Nacionalidad } & Chilena & 1379 & 97,8 \\
\hline & Extrajera & 31 & 2,2 \\
\hline \multirow[t]{4}{*}{ Origen Étnico } & Mapuche & 133 & 9,4 \\
\hline & Rapanui & 2 & 0,1 \\
\hline & No Pertenece & 867 & 61,5 \\
\hline & No lo sabe & 408 & 28,9 \\
\hline \multirow[t]{4}{*}{ Cursos } & $5^{\circ}$ Básico & 376 & 26,7 \\
\hline & $6^{\circ}$ Básico & 339 & 24 \\
\hline & $7^{\circ}$ Básico & 379 & 26,9 \\
\hline & $8^{\circ}$ Básico & 316 & 22,4 \\
\hline \multirow[t]{3}{*}{ Dependencia Administrativa } & Municipal & 431 & 30,6 \\
\hline & Subvencionado & 564 & 40 \\
\hline & Privado & 415 & 29,4 \\
\hline \multirow[t]{6}{*}{ Establecimiento Educacional } & Municipal 1 & 201 & 14,3 \\
\hline & Municipal 2 & 230 & 16,3 \\
\hline & Subvencionado 1 & 276 & 19,6 \\
\hline & Subvencionado 2 & 287 & 20,4 \\
\hline & Pagado 1 & 197 & 14 \\
\hline & Pagado 2 & 219 & 15,5 \\
\hline
\end{tabular}

\subsection{INSTRUMENTO}

Se utilizó el Cuestionario de Convivencia Escolar para la No Violencia (CENVI). Dicho instrumento explora la percepción de los estudiantes en relación a la violencia escolar y la gestión de la convivencia. De esta forma, el modelo propuesto presenta dos factores, con cinco y tres dimensiones respectivamente (ver tabla 2 ). 
Tabla 2: Modelo Cuestionario CENVI

\begin{tabular}{|c|c|l|l|}
\hline \multicolumn{2}{|l|}{ Factores } & Dimensiones & Abreviatura \\
\hline \multirow{4}{*}{ Factor 1 } & 1 & Violencia Verbal & V. Verbal \\
\cline { 2 - 5 } & 2 & Violencia Física-Conductual & V. Física-Conductual \\
\cline { 2 - 5 } & 3 & Violencia Social de exclusión & V. Social-Exclusión \\
\cline { 2 - 5 } & 4 & Violencia por Medios Tecnológicos & V. Medios Tecnológicos \\
\cline { 2 - 5 } & 5 & Violencia de profesores a estudiantes & V. Profesor a Estudiante \\
\hline \multirow{4}{*}{ Factor 2 } & 1 & Formación para la No Violencia & F. No Violencia \\
\cline { 2 - 5 } & 2 & Gestión para la No Violencia & G. No Violencia \\
\cline { 2 - 5 } & 3 & Participación & Participación \\
\hline
\end{tabular}

El primer factor denominado Tipos de Violencia Escolar (TVE), explora las percepciones de los estudiantes en relación a las formas de manifestación de la violencia en contextos escolares. Con un total de 49 ítems, este factor está compuesto por cinco dimensiones que abordan las percepciones sobre: 1) Violencia verbal: agresiones por medio de palabras, como insultos, amenazas para ejercer dominio sobre la víctima o humillarla; 2) Violencia física: agresiones físicas como empujones, tirones de pelo, pellizcos, apretones, golpes de puño o con objetos. Violencia física directa, es cuando la agresión se aplica sobre cualquier parte del cuerpo de la víctima, y es violencia física indirecta, cuando se causa daño sobre sus pertenencias o materiales de trabajo; 3) Exclusión social: actos de discriminación o rechazo, por rendimiento académico, nacionalidad, diferencias culturales o raciales, o el aspecto físico; 4) Violencia por medios tecnológicos: comportamientos violentos donde las agresiones son principalmente a través del teléfono móvil e Internet, utilizando fotos, grabaciones o mensajes con la intención de causar daño y; 5) Violencia de profesores a estudiantes: el profesor es quien agrede al estudiante, ya sea verbalmente por medio de insultos, burlas o apodos; físicamente por medio de zamarreos para llamarle la atención o sacarlo de la sala de clases y; discriminando por razones académicas, culturales o físicas.

El segundo factor denominado Gestión de la Convivencia (GC), explora las percepciones de los estudiantes en relación a aspectos centrales de gestión establecidos por la política pública de convivencia y la forma en que son implementados por la escuela: formación integral, aseguramiento de entornos libres de malos tratos y participación y vida democrática. Este factor está compuesto por 30 ítems que se agrupan en tres dimensiones: 1) Formación para la No violencia: refiere a prácticas para la reflexión y educación enmarcadas en el diálogo, respeto y la aceptación legítima del otro, con la finalidad de disminuir el riesgo de situaciones de violencia; 2) Gestión para la no violencia: se relaciona con la construcción y cumplimiento de normas de convivencia para el control y la sanción de la violencia. Esta dimensión está centrada en la comunicación fluida y oportuna entre familia y escuela y; 3) Participación: refiere a las prácticas y desarrollo de acciones orientadas a la integración de los miembros de la comunidad educativa, para contribuir a la construcción de espacios seguros y libres de malos tratos que afecten la sana convivencia. 
La construcción del cuestionario CENVI consideró las orientaciones de dos encuestas revisadas en el contexto nacional e internacional: Para el diseño del primer factor, se tomó como orientación el Cuestionario de Violencia Escolar [CUVE] (Álvarez et al., 2006; Álvarez et al., 2011; Álvarez-García et al., 2013) que cuenta con una aplicación en Chile (Guerra et al., 2011). El instrumento se centra en la percepción de los estudiantes sobre la frecuencia de aparición de diferentes tipos de violencia escolar, protagonizados por estudiantes y profesores. Para el diseño del segundo factor se tomó como orientación el Cuestionario de Convivencia Escolar [CCE] (Chaparro, et al., 2012). El instrumento explora las dimensiones de inclusión, democracia y paz. Los ítems de este factor en el cuestionario CENVI, se ajustaron a los estándares de formación y convivencia entregados por MINEDUC (2014).

El cuestionario CENVI mide variables categóricas por medio de una escala de autorespuesta de tipo Likert, donde los encuestados pueden opinar sobre la frecuencia en que se manifestarían los enunciados expuestos en cada ítem. Son cuatro opciones de respuesta que van de 0 a 3: $0=$ Nunca, $1=$ Pocas veces, $2=$ Frecuentemente y, $3=$ Siempre. Para el factor tipos de violencia escolar, a mayor puntaje mayor expresión de violencia. En el caso del factor gestión de la convivencia, a mayor puntaje mayores acciones y prácticas favorables para la gestión de la no violencia.

\subsection{PROCEDIMIENTO Y PLAN DE ANÁLISIS}

El proceso de construcción y validación del instrumento, se realizó en las siguientes fases:

a) Levantamiento de ítems: Es la etapa en que se elaboraron los reactivos del cuestionario CENVI. Esto implicó identificar los principales elementos y conceptos que subyacen en los diagnósticos nacionales sobre violencia escolar y los ejes en que se fundamenta la política pública sobre convivencia.

b) Adaptación lingüística: Corresponde a la revisión de los ítems seleccionados del instrumento CUVE (Álvarez et al., 2011) y del CCE (Chaparro et al., 2012) que serían incorporados al instrumento CENVI. Lo anterior con la finalidad de identificar las dificultades de comprensión del idioma en España y México para su variante en Chile.

c) Validación de contenido (Hernández, Fernández y Baptista, 2010): el contenido del instrumento fue validado vía revisión de tres jueces expertos en el área, pertenecientes a la Universidad Católica de Temuco (UCT), a la Pontificia Universidad Católica de Chile (PUC) y, un experto integrante de la Red Latino Americana de Convivencia Escolar (RLCE). Los jueces presentaron sugerencias de modificaciones a la estructura del instrumento, conceptos de base a los factores y dimensiones, extensión o suficiencia de las dimensiones y, a la redacción y pertinencia evolutiva para la comprensión de los ítems.

d) Análisis estadístico de prueba piloto: Esta etapa contribuyó a validar la comprensión de los ítems elaborados, la adaptación lingüística y adaptación de contenido. Primero se aplicó el instrumento a 4 estudiantes de $5^{\circ}$ y $8^{\circ}$ básico, con la finalidad de obtener la opinión de personas cuyas características correspondieran a la muestra final del estudio. Luego el pilotaje se realizó con 47 estudiantes de $5^{\circ}$ y $8^{\circ}$ año básico de un establecimiento particular subvencionado de la ciudad de 
Temuco. La prueba recogió dudas y comentarios de los estudiantes, que implicó modificaciones en la aplicación definitiva.

e) Aplicación general del instrumento: Consideró previamente la negociación de acceso al campo, el consentimiento de los directores y asentimiento de los padres y estudiantes. Respecto al control de variables intervinientes, se cauteló la influencia de elementos distractores, asegurando que los horarios, espacios y condiciones fueran óptimas para la aplicación del instrumento.

f) Análisis Factorial Confirmatorio (AFC): Para su análisis se consideró la escala de medida ordinal, de modo que se utilizó la matriz de correlaciones policóricas y el método de estimación de Robust unweighted least squares (ULSMV). La matriz de correlaciones policóricas se recomienda si la totalidad de las variables es ordinal o algunas son ordinales y otras dicotómicas. La escala Likert del instrumento es ordinal, por lo tanto se utilizó esta matriz de correlación (Finney y Distefano, 2006). Considerando estas características, para evaluar el ajuste del modelo se emplearon: 1) El estadístico Chi-cuadrado, teniendo en cuenta que el valor de $\chi 2$ es muy sensible a pequeñas desviaciones del modelo hipotetizado cuando se trabaja con grandes muestras (Jöreskog, 1970; Jöreskog; Sörbom, 1993), se considera adecuado cuando la proporción de la división de los grados de libertad en relación al índice no es superior a 3:1 ( $\mathrm{X}^{2}$ :df ratio <3:1) (Kline, 2015);2) El error cuadrático medio de aproximación (RMSEA); 3) El índice Tucker-Lewis (TLI) y; 4) El índice de bondad de ajuste comparativo (CFI) como medidas de ajuste incremental. Hu y Bentler (2009) consideran valores aceptables del CFI, TLI superiores a .90, considerándose excelentes valores superiores a .95. Por último, el modelo será considerado con buen ajuste si el RMSEA es inferior a .05 (Batista-Foguet, Coenders, Alonso, 2004; Brown, 2012).

g) El análisis final de confiabilidad se realizó por medio del coeficiente de alfa de Cronbach. Los análisis se realizaron usando los programas SPSS en su versión 20 y Mplus en su versión 6.11 (Muthén y Muthén, 2007).

\section{RESULTADOS}

\subsection{VALIDEZ DE CONTENIDO}

a) Elaboración de ítems: La etapa permitió una primera versión del cuestionario CENVI compuesta por 98 ítems. Un 60\% de los reactivos se agrupó en los tipos de violencia, que en parte se tomaron del instrumento CUVE. El cuarenta por ciento de los ítems se agrupó en la gestión de la convivencia, que fueron elaborados principalmente a partir de los estándares indicativos de desempeño entregados por MINEDUC en 2014 y en menor medida en base a orientaciones de lo observado en el instrumento CCE.

b) Ajuste Lingüístico: Esta etapa examinó únicamente los ítems seleccionados del instrumento CUVE y los tomados como orientación del CCE, que luego formarían parte del cuestionario CENVI. La etapa implicó cambios en 2 ítems que incluían términos cuya comprensión difiere en idioma español de un país a otro y 12 que incluían conceptos comprensibles, pero no usados comúnmente en Chile. 
c) Revisión de jueces expertos: Implicó modificaciones en la 'Estructura del instrumento', mejorando la coherencia interna de los factores y sus dimensiones en relación a la pertinencia de los conceptos explorados. En los 'Conceptos de base a los factores y dimensiones', existió cambios a partir de sugerencias a los términos empleados para evitar aplicaciones teóricamente erróneas. En la 'Suficiencia teórica de las dimensiones', hubo ajustes al identificar reactivos que exploraban aspectos no esenciales en cada dimensión. Por último, en la 'Comprensión y pertinencia evolutiva de los reactivos', las modificaciones se produjeron al revelar aquellos que presentaban dificultad para su comprensión teórica, cautelando la pertinencia evolutiva en base a la edad de los participantes. Al finalizar la etapa de revisión de jueces expertos, el constructo que inicialmente tenía 98 ítems se redujo a 79.

\subsection{ANÁLISIS ESTADÍSTICOS DE LA APLICACIÓN PILOTO}

El análisis de confiabilidad mostró valores alfa de Cronbach que oscilaron entre .810 y .903 (ver tabla 3). Se realizaron pruebas de eliminación aleatoria de ítems, obteniendo como resultado la pérdida de consistencia interna en la dimensión correspondiente al reactivo que se eliminaba. Si bien la aplicación piloto no recibió comentarios pertinentes a la comprensión de los ítems, se cambió la orientación del número 61, que hasta el momento era inversa al resto de los reactivos del factor al que pertenece.

Tabla 3: Índice de confiabilidad aplicación piloto cuestionario CENVI

\begin{tabular}{|c|l|c|c|c|c|c|}
\hline \multirow{2}{*}{ Factor } & \multicolumn{1}{|c|}{ Dimensiones } & $\begin{array}{c}\text { Alfa de } \\
\text { Cronbach }\end{array}$ & $\begin{array}{c}\text { Media } \\
\%\end{array}$ & $\begin{array}{c}\text { Varianza } \\
\%\end{array}$ & $\begin{array}{c}\text { Desviación } \\
\text { Típica } \%\end{array}$ & $\begin{array}{c}\text { Ítems por } \\
\text { dimensión }\end{array}$ \\
\hline \multirow{4}{*}{$\begin{array}{c}\text { Factor } 1 \\
\text { TVE }\end{array}$} & V. Verbal & 0,810 & 8,89 & 25,401 & 5,040 & 10 \\
\cline { 2 - 7 } & V. Física-Conductual & 0,843 & 8,89 & 29,749 & 5,454 & 11 \\
\cline { 2 - 7 } & V. Social-Exclusión & 0,838 & 7,36 & 30,540 & 5,526 & 10 \\
\cline { 2 - 7 } & V. Medios Tecnológicos & 0,842 & 5,38 & 22,720 & 4,767 & 9 \\
\cline { 2 - 7 } & V. Profesor a Estudiante & 0,830 & 6,57 & 32,467 & 5,698 & 9 \\
\hline \multirow{3}{*}{$\begin{array}{c}\text { Factor } 2 \\
\text { GC }\end{array}$} & F. No Violencia & 0,875 & 18,77 & 72,227 & 8,499 & 12 \\
\cline { 2 - 7 } & G. No Violencia & 0,845 & 17,77 & 52,488 & 7,245 & 10 \\
\cline { 2 - 7 } & Participación & 0,903 & 12,66 & 51,838 & 7,200 & 8 \\
\hline
\end{tabular}

\subsubsection{Análisis factorial confirmatorio (AFC)}

Análisis previos al AFC: Los resultados de la Medida de adecuación muestral de KaiserMeyer-Olkin (KMO) y de esfericidad de Bartlett, indican que los datos pueden ser reducidos en factores, $\mathrm{KMO}=0,963$; Barlett $\mathrm{p}<0,001$. El modelo propuesto se puede ver en la tabla 4 . 
Tabla 4: Modelo propuesto para el análisis de la estructura del cuestionario CENVI

\begin{tabular}{|c|c|l|l|}
\hline Factores & & Dimensiones & Ítems \\
\hline \multirow{4}{*}{$\begin{array}{c}\text { Factor } 1 \\
\text { TVE }\end{array}$} & 1 & V. Verbal & $1,2,3,4,5,6,7,8,9,10$ \\
\cline { 2 - 4 } & 2 & V. Física-Conductual & $11,12,13,14,15,16,17,18,19,20,21$ \\
\cline { 2 - 4 } & 3 & V. Social-Exclusión & $22,23,24,25,26,27,28,29,30,31$ \\
\cline { 2 - 4 } & 4 & V. Medios Tecnológicos & $32,33.34,35,36,37,38,39,40$ \\
\cline { 2 - 4 } & 5 & V. Profesor a Estudiante & $41,42,43,44,45,46,47,48,49$ \\
\hline \multirow{3}{*}{$\begin{array}{c}\text { Factor } 2 \\
\text { GC }\end{array}$} & 1 & F. No Violencia & $50,51,52,53,54,55,56,57,58,59,60,61$ \\
\cline { 2 - 4 } & 2 & G. No Violencia & $62,63,64,65,66,67,68,69,70,71$ \\
\cline { 2 - 4 } & 3 & Participación & $72,73,74,75,76,77,78,79$ \\
\hline
\end{tabular}

Considerando todos los ítems de la escala, y que éstos presentan una escala de medida ordinal, se ha procedido a utilizar la matriz de correlaciones policóricas y el método de estimación de Mínimos Cuadrados Parciales (ULSMV). El modelo propuesto presenta un buen ajuste $\mathrm{X}^{2}=7993,75 ; \mathrm{Df}=2993 ; \mathrm{CFI}=0,912$; TLI=0,91; RMSEA=0,033

Los índices señalados indican un buen ajuste al modelo de 2 factores que explican las diferentes percepciones que los estudiantes tienen sobre la violencia en la escuela, y la gestión de la convivencia desarrollada por la institución educativa.

\subsubsection{Análisis de confiabilidad}

El análisis estadístico de los datos ha mostrado que el instrumento posee buenos indicadores de confiablidad, arrojando valores $\alpha$ entre .853 y .902 para las dimensiones. El alfa total del factor 1 es .962 y del factor 2 es .944 . Se puede apreciar los valores para cada dimensión en la tabla 5.

Tabla 5: Índices de confiabilidad para el cuestionario CENVI

\begin{tabular}{|c|l|c|c|c|c|c|}
\hline \multirow{2}{*}{ Factores } & \multicolumn{1}{|c|}{ Dimensiones } & $\begin{array}{c}\text { Alfa de } \\
\text { Cronbach }\end{array}$ & $\begin{array}{c}\text { Media } \\
\%\end{array}$ & $\begin{array}{c}\text { Varianza } \\
\%\end{array}$ & $\begin{array}{c}\text { Desviación } \\
\text { Típica } \%\end{array}$ & $\begin{array}{c}\text { Ítems por } \\
\text { dimensión }\end{array}$ \\
\hline \multirow{4}{*}{$\begin{array}{c}\text { Factor } 1 \\
\text { TVE }\end{array}$} & V. Verbal & 0,810 & 13,39 & $35,5 \%$ & 5,929 & 10 \\
\cline { 2 - 7 } & V. Física-Conductual & 0,886 & 10,21 & $43,3 \%$ & 10,821 & 11 \\
\cline { 2 - 7 } & V. Social-Exclusión & 0,902 & 10,93 & $46,5 \%$ & 7,236 & 10 \\
\cline { 2 - 7 } & V. Medios Tecnológicos & 0,886 & 10,21 & $43,0 \%$ & 6,821 & 9 \\
\cline { 2 - 7 } & V. Profesor a Estudiante & 0,911 & 5,76 & $39,2 \%$ & 5,901 & 9 \\
\hline \multirow{3}{*}{$\begin{array}{c}\text { Factor } 2 \\
\text { GC }\end{array}$} & F. No Violencia & 0,853 & 7,49 & $50,6 \%$ & 5,633 & 12 \\
\cline { 2 - 7 } & G. No Violencia & 0,896 & 18,14 & $54,1 \%$ & 8,532 & 10 \\
\cline { 2 - 7 } & Participación & 0,890 & 18,20 & $54,9 \%$ & 7,355 & 8 \\
\hline
\end{tabular}




\section{DISCUSIÓN}

El estudio cumple el objetivo de entregar un instrumento que evalúa de manera conjunta y desde la perspectiva de los estudiantes, los tipos de violencia manifestados en la escuela y la gestión de la convivencia escolar. El factor 1 del instrumento CENVI, llamado tipos de violencia escolar, quedó estructurado por las dimensiones: Violencia verbal, Violencia conductual, Violencia social de exclusión, Violencia por medios tecnológicos y Violencia profesor alumno. Su estructura cumple el objetivo de explorar los tipos de violencia manifestados en la escuela. El factor 2 del instrumento CENVI, llamado gestión de la convivencia, está compuesto por las dimensiones: Formación para la no violencia, Gestión para la no Violencia y, Participación. En su elaboración se cauteló que el levantamiento de cada ítem fuera coherente con los estándares de convivencia escolar entregados por MINEDUC (2014). El factor 2 del cuestionario CENVI cumple el objetivo de medir desde la percepción de los estudiantes, los aspectos de gestión de la convivencia que se desarrolla en la escuela.

La validación teórica de jueces expertos aportó información relevante para la toma de decisiones que implicó modificación en la redacción de ítems, reubicación de estos en relación a su dimensión y la eliminación de algunos reactivos. Lo anterior pudo influir en que el modelo propuesto ajustara adecuadamente en el AFC.

Desde el Análisis Factorial Confirmatorio, el modelo propuesto en el cuestionario CENVI con 2 factores, compuestos por 5 y 3 dimensiones respectivamente, ha presentado un buen ajuste. Aportando con esto al soporte teórico que subyace a la construcción del instrumento.

A diferencia de los instrumentos existentes en Chile, el cuestionario CENVI reporta datos tanto de violencia escolar como de gestión de la convivencia. Esto se constituye en un insumo importante, ya que el factor 2 (gestión de la convivencia), facilita ampliamente la toma de decisiones para la intervención, en tanto sus dimensiones están alineadas con los estándares actuales entregados por MINEDUC (2014).

Todo lo anterior aporta a mejorar la convivencia escolar cuando ésta se encuentra deteriorada por elementos disfuncionales (Giancola y Bear, 2003) afectando el normal desarrollo de las actividades curriculares (Sánchez, Rivas y Trianes, 2006). Así, con la información reportada por el instrumento, las autoridades de la escuela podrán tomar acciones preventivas frente a la violencia escolar, que entre otras cosas genera inadaptaciones sociales y patologías, tanto en víctimas como agresores (Kochenderfer y Ladd, 1996; Eisenberg, Neumark-Sztainer y Perry, 2003; Fekkes et al., 2006; Hinduja y Patchin, 2010).

En conclusión, la validación del instrumento elaborado muestra que el modelo propuesto se aproxima correctamente a lo que desea medir, evidenciando una apropiada calidad psicométrica, cuyos índices de confiabilidad dan garantías suficientes para ampliar su uso en el contexto nacional.

Futuras investigaciones debiesen considerar los siguientes elementos: a) muestreos aleatorios que permitan un menor sesgo; b) ampliar el rango de los cursos integrados en el estudio ${ }^{1}$; c) profundizar en las causas de la violencia manifiesta, lo que permitiría fortalecer las acciones de mejora; d) incluir una validación concurrente, que entregue datos de la correlación del instrumento con otros validados previamente y; e) un test-retest para determinar la estabilidad temporal del instrumento.

Ampliar el rango de cursos implicaría probar un modelo alternativo que excluya la dimensión de violencia por medios tecnológicos, ya que los datos confirman que a menor edad, menor prevalencia de ésta. 
La investigación puede considerar en el futuro, elementos relativos a la diferencia del hecho de la violencia por género, por origen cultural y nivel sociocultural de pertenencia. Además, que debiera ser complementado por estudios paralelos de orden cualitativo, con el fin de comprender las razones por las cuales se desarrolla o no algún tipo de violencia o la percepción de la misma desde el sistema educativo o del grupo de pertenencia de los estudiantes.

\section{REFERENCIAS BIBLIOGRÁFICAS}

Albaladejo, N. (2011). Evaluación de la violencia escolar en educación infantil y primaria. (Tesis Doctoral, Universidad de Alicante, España) Recuperada de https://rua.ua.es/dspace/ bitstream/10045/24847/1/Tesis_Albaladejo.pdf

Álvarez-García, D., Núñez, J. y Dobarro, A. (2013). Cuestionarios para evaluar la violencia escolar en Educación Primaria y en Educación Secundaria: CUVE3-EP y CUVE3-ESO. Apuntes de Psicología, vol. 31 (2), 191-202. Recuperado el 05 de enero de 2017 desde http://www. apuntesdepsicologia.es/index.php/revista/article/download/322/296

Álvarez-García, D., Núñez, J., Rodríguez, C., Álvarez, L., y Dobarro, A. (2011). Propiedades psicométricas del Cuestionario de Violencia Escolar-Revisado (CUVE-R). Revista de Psicodidáctica, vol. 16 (1), 59-83. Recuperado el 06 de enero de 2017 desde http://www.ehu. eus/ojs/index.php/psicodidactica/article/download/1146/786

Álvarez, L., Álvarez, D., Gonzáles-Castro, P. Núñez, J. y Gonzáles-Pienda, J. (2006) Evaluación de los comportamientos violentos en los centros educativos. Psicothema, vol. 18 (4), 686-695. Recuperado el 06 de enero de 2017 desde http://dspace.sheol.uniovi.es/dspace/ bitstream/10651/26662/1/Psicothema.2006.18.4.686-95.pdf

Batista-Foguet, J.M., Coenders, G. y Alonso, J. (2004). Análisis Factorial Confirmatorio. Su utilidad en la validación de cuestionarios relacionados con la salud. Medicina Clínica, vol. 122 (1), 2127. Recuperado el 05 de enero de 2017 de http://www.elsevier.es/es-revista-medicina-clinica-2articulo-analisis-factorial-confirmatorio-su-utilidad-13057542

Berger, C., y Lisboa, C. (2009). Violencia escolar: estudios y posibilidades de intervención en Latinoamérica. Santiago de Chile: Editorial Universitaria.

Brown, T. (2012). Confirmatory factor analysis for Applied Research. New York: Guilford Press.

Buelga, S., Cava, M.J. y Musitu, G. (2012). Validación de la Escala de Victimización entre Adolescentes a través del Teléfono Móvil y de Internet. Revista Panamericana de Salud Pública, vol. 32 (1), 36-42. Recuperado el 05 de enero de 2017 desde http://www.scielosp.org/scielo. php?script=sci_arttext\&pid=S1020-49892012000700006

Cava, M.J., Buelga, S., Musitu, G. y Murgui, S. (2010). Violencia Escolar entre Adolescentes y sus Implicaciones en el Ajuste Psicosocial: Un Estudio Longitudinal. Revista Psicodidáctica, vol. 15 (1) 21-24. Recuperado el 05 de enero de 2017 desde http://www.redalyc.org/articulo. oa? $\mathrm{id}=17512968002$

Chaparro, A., Caso, J., Fierro, M. y Díaz C. (2015). Desarrollo de un instrumento de evaluación basado en indicadores de convivencia escolar democrática, inclusiva y pacífica. Perfiles Educativos, vol. 37 (149), 20-41. Recuperado el 06 de enero de 2017 desde http://132.248.192.201/seccion/ perfiles/2015/n149a2015/mx.peredu.2015.n149.p20-41.pdf

Chaparro, A., Caso, J., Diaz, C. y Urias, E. (2012). Instrumentos para el Autodiagnóstico e Intervención en Escuelas Basados en Indicadores de Convivencia democrática, inclusiva y no violenta. Ensenada, México: Universidad Autónoma de Baja California. Recuperado el 06 de enero de 2017 desde http://uee.uabc.mx/docs/reportesTecnicos/2012/UEERT12-004.pdf

Cid, A. (2004). El Clima Escolar como Factor de Calidad en los Centros de Educación Secundaria de 
la Provincia de Ourense. Su Estudio desde la Perspectiva de la Salud. Revista de investigación Educativa, vol. 22 (1) 113-144

Consejo Nacional para el Control de Estupefacientes. (2006). Sexto estudio nacional de drogas en población escolar de Chile: $8^{\circ}$ básico a $4^{\circ}$ medio: Informe Final. Recuperado desde http://www. senda.gob.cl/media/estudios/PE/2005_Sexto_EstudioEscolar.pdf

Dobarro, A., Álvarez García, D., Núñez, J.C. (2014). CUVE3: Instrumentos para evaluar la violencia escolar. Revista de Psicología, vol. 5 (1), 487-492. doi: 10.17060/ijodaep.2014.n1.v5.710

Eisenberg, M.E., Neumark-Sztainer, D. y Perry, CL. (2003). Peer Harassment, School Connectedness, and Academic Achievement. Journal of School Health, vol. 73 (8), 311-316. doi: 10.1111/ j.1746-1561.2003.tb06588.x

Fekkes, M., Pijpers, F., Miranda, A., Vogels, T., Verloove - Vanhorick, S. (2006). Do Bullied Children Get Ill, or Do Ill Children Get Bullied? A Prospective Cohort Study on the Relationship Between Bullying and Health-Related Symptoms. American Academy of Pediatrics, vol. 117, (5), 15681574. doi:10.1542/peds.2005-0187

Finney, S. y Distefano, C. (2006). Non-normal and categorical data in structural equation modeling. Structural equation modeling: A second course, (pp. 269-314).

Giancola, S. y Bear, G. (2003). Face fidelity: Perspectives from a local evaluation of the Safe Schools/ Healthy Students Initiative. Psychology in the Schools, vol. 40 (5), 515-529.

Guerra, C., Álvarez-García, D., Dobarro, A., Núñez, J., Castro, L. y Vargas, J. (2011). Violencia Escolar en Estudiantes de Educación Secundaria de Valparaíso (Chile): Comparación con una muestra Española. Revista iberoamericana de psicología y salud, vol. 2 (1), 75-98. Recuperado el 05 de enero de 2017 desde https://dialnet.unirioja.es/servlet/articulo?codigo=3348577

Guerra, C., Castro, L. y Vargas, J. (2011). Examen psicométrico del Cuestionario de clima social del Centro Escolar en estudiantes chilenos. Psicothema, vol. 23, (1), 140-145. Recuperado el 06 de enero de 2017 desde http://www.unioviedo.es/reunido/index.php/PST/article/view/9065

Hernández, R., Fernández, C. y Baptista, P. (2010). Metodología de la investigación. México: Mc Graw Hill.

Hinduja, S. y Patchin, JW. (2010). Bullying, Cyberbullying, and Suicide. Archives of Suicide Research, vol.14 (3), 206-221. DOI: 10.1080/13811118.2010.494133

Hu, Li-tze y Bentler, Peter M. (1999). Cutoff criteria for fit indexes in covariance structure analysis: Conventional criteria versus new alternatives. Structural Equation Modeling: A Multidisciplinary Journal, vol. 6, (1), 1-55 doi:10.1080/10705519909540118.

Joreskog, K. (1970). A general method for analysis of covariance structures. Biometrika, vol. 57 (2), 239-251.

Jöreskog, K., Sörbon, D. (1993) LISREL 8: Structural equation modeling with the SIMPLIS command language. Scientific Software International.

Kline, R. (2015). Principies and practice of structural equation modeling. New York: Guilford.

Kochenderfer, B. y Ladd, G. (1996). Peer Victimization: Cause or Consequence of School Maladjustment? Child Development, vol. 67 (4) 1305-1317.

Lecannelier, F., Varela, J., Rodríguez, J., Hoffmann, M., Flores, F. y Ascanio, L. (2011). Validación del Cuestionario de Maltrato entre Iguales por Abuso de Poder (MIAP) para escolares. Revista médica de Chile, vol. 139 (4), 474-479. doi:10.4067/S0034-98872011000400009

Ley 20370. Diario Oficial de la República de Chile, Santiago, Chile. 12 de septiembre de 2009. Recuperado el 05 de enero de 2017 desde http://bcn.cl/1m0i2

Ley 20536. Diario Oficial de la República de Chile, Santiago, Chile. 17 de septiembre de 2011. Recuperado de http://bcn.cl/1lzbx

López, V., Bilbao, M. y Rodriguez, J. (2012). La sala de clases sí importa: incidencia del clima de aula sobre la percepción de intimidación y victimización entre escolares. Universitas Psychologica, vol. 11 (1), 91-101. Recuperado el 05 de enero de 2017 desde http://www.redalyc.org/articulo. oa? $\mathrm{id}=64723234008$ 
Magendzo, A., Toledo- M. y Gutiérrez, V. (2013). Descripción y análisis de la Ley sobre Violencia Escolar ( $\mathrm{N}^{\circ} 20.536$ ): dos paradigmas antagónicos. Estudios pedagógicos (Valdivia), vol. 39 (1), 377-391. doi: 10.4067/S0718-07052013000100022

Marín-Martínez, A, y Reidl, L. (2013). Validación psicométrica del cuestionario "Así nos llevamos en la escuela" para evaluar el hostigamiento escolar (bullying) en primarias. Revista mexicana de investigación educativa, vol. 18 (56), 11-36. Recuperado en 05 de enero de 2017 desde http:// www.scielo.org.mx/scielo.php?script=sci_arttext\&pid=S1405-66662013000100002\&lng=es\& tlng=es.

Ministerio de Educación (2014). Estandares indicativos de desempeño para los establecimientos educacionales y sus Sostenedores. Santiago de Chile: MINEDUC.

Ministerio de Educación (2015). Política nacional de convivencia escolar. Santiago de Chile: MINEDUC.

Instituto de Evaluación y Asesoramiento Educativo (2005). Primer estudio nacional de convivencia escolar. Recuperado el 14 de marzo de 2017 de http://www.idea-educa.cl/descargas/ convivencia_escolar.pdf.

Ministerio Nacional de Educación de Chile y Universidad Alberto Hurtado (2006). Principales resultados del estudio de violencia en el ámbito escolar. Recuperado de http://www. seguridadpublica.gov.cl/filesapp/pres_ppt.pdf

Murillo, J. (2011). Mejora de la eficacia escolar en Iberoamérica. Revista Iberoamericana de educación, (55), 49-83. Recuperado el 05 de enero de 2017 desde rieoei.org/rie55a02.pdf

Muthén, L. y Muthén, B. (2007). Mplus User's Guide (Sixth Edition). Los Angeles, CA: Muthén \& Muthén.

Sánchez, A. Rivas, M. y Trianes, M (2006). Eficacia de un programa de intervención para la mejora del clima escolar, Electronic journal of research in educational psychology, vol. 4 (2)353-370.

Sánchez, C. y Cerezo, F. (2011). Estatus social de los sujetos implicados en Bullying. Elementos para la mejora de la convivencia en el aula. Revista Española de Orientación y Psicopedagogía, vol. 22 (2) 137-149. Recuperado el 05 de enero de 2017 desde http://revistas.uned.es/index.php/reop/ article/download/62/52 


\section{ANEXOS}

Anexo A: Cuestionario CENVI

\section{CUESTIONARIO CENVI CONVIVENCIA ESCOLAR PARA LA NO VIOLENCIA}

Este cuestionario es anónimo, sin embargo es necesario saber algunas características de los estudiantes que lo responden. A continuación deberás marcar con una $\mathbf{X}$ sobre el cuadrado de la alternativa que corresponda a tu realidad o escribiendo la respuesta según sea el caso.

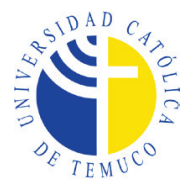

\section{1.- Indica tu edad}

\section{2.- Indica tu género}

\begin{tabular}{|l|l|l|l|}
\hline Hombre & $\square$ Mujer & & \\
\hline
\end{tabular}

3.- Nacionalidad

\begin{tabular}{|l|l|l}
$\square$ Chilena & $\square$ Extranjera & \\
\hline
\end{tabular}

4.- ¿Perteneces a algún pueblo originario?

\begin{tabular}{|l|l|l|l}
\hline$\square$ Mapuche & $\square$ Aymara & $\square$ Rapanui & $\square$ Quechua \\
\hline$\square$ Atacameño & $\square$ Coya & $\square$ Kawashkar & $\square$ Yagan \\
\hline$\square$ Diaguita & $\square$ NO pertenezco & $\square$ NO lo sé & \\
\hline
\end{tabular}

5- ¿En qué curso te encuentras actualmente?

\begin{tabular}{|l|l|l|l}
$\square 5^{\circ}$ básico & $\square 6^{\circ}$ básico & $\square 7^{\circ}$ básico & $\square 8^{\circ}$ básico \\
\hline
\end{tabular}

6.- ¿Cuál es la dependencia de tu establecimiento educacional?

Municipal

7.- ¿Cuál es el nombre de tu establecimiento educacional? 
Te presentamos una serie de preguntas acerca de situaciones que ocurren a veces en las escuelas, liceos o colegios, para que tú respondas cómo ves la realidad de tu colegio. Puedes responder muy honestamente pues el cuestionario es anónimo (no va con tu nombre). Tus respuestas son muy importantes para que tu colegio progrese, si es que tiene algunos problemas.

Para contestar el cuestionario, deberás señalar qué tanto ocurre cada situación en tu colegio, marcando una $\mathbf{X}$ en el cuadrado que corresponda, siguiendo esta clave:
$\mathbf{A}=$ Siempre
$\mathbf{B}=$ Frecuentemente
$\mathbf{C}=$ Pocas veces
$\mathbf{D}=$ Nunca

\begin{tabular}{|c|c|c|c|c|c|}
\hline & & $\mathbf{A}$ & B & $\mathbf{C}$ & D \\
\hline 1. & $\begin{array}{l}\text { Hay estudiantes que extienden (echan a correr) rumores negativos } \\
\text { o mal intencionados acerca de otros compañeros/as. }\end{array}$ & $\square$ & $\square$ & $\square$ & $\square$ \\
\hline 2. & $\begin{array}{l}\text { Algunos estudiantes ponen sobrenombres molestos a sus } \\
\text { compañeros/as. }\end{array}$ & $\square$ & $\square$ & $\square$ & $\square$ \\
\hline 3. & $\begin{array}{l}\text { Ciertos estudiantes hablan mal o "pelan" a otros compañeros/as } \\
\text { cuando estos no están. }\end{array}$ & $\square$ & $\square$ & $\square$ & $\square$ \\
\hline 4. & $\begin{array}{l}\text { Hay estudiantes que burlan o insultan verbalmente a compañeros/ } \\
\text { as por sus características físicas o su forma de ser. }\end{array}$ & $\square$ & $\square$ & $\square$ & $\square$ \\
\hline 5. & $\begin{array}{l}\text { Hay compañeros/as que avergüenzan a otros haciéndole burlas } \\
\text { con la persona que le gusta, para hacerles quedar en ridículo. }\end{array}$ & $\square$ & $\square$ & $\square$ & $\square$ \\
\hline 6. & $\begin{array}{l}\text { Hay estudiantes que ofenden a algunos compañeros/as burlándose } \\
\text { de las características de su familia. }\end{array}$ & $\square$ & $\square$ & $\square$ & $\square$ \\
\hline 7. & $\begin{array}{l}\text { Hay estudiantes que ofenden verbalmente a compañeros/as por } \\
\text { ser indígenas o extranjeros. }\end{array}$ & $\square$ & $\square$ & $\square$ & $\square$ \\
\hline 8. & $\begin{array}{l}\text { En el colegio algunos estudiantes amenazan a otros de palabra } \\
\text { para causarles miedo u obligarles a hacer cosas. }\end{array}$ & $\square$ & $\square$ & $\square$ & $\square$ \\
\hline 9. & $\begin{array}{l}\text { Algunos estudiantes esperan a sus compañeros/as a la salida del } \\
\text { colegio para seguir molestándolos. }\end{array}$ & $\square$ & $\square$ & $\square$ & $\square$ \\
\hline 10. & $\begin{array}{l}\text { Hay compañeros/as que insultan verbalmente o hacen bromas a } \\
\text { algunos profesores para hacerles quedar en ridículo. }\end{array}$ & $\square$ & $\square$ & $\square$ & $\square$ \\
\hline 11. & Hay estudiantes que le pegan a compañeros/as dentro del colegio. & $\square$ & $\square$ & $\square$ & $\square$ \\
\hline 12. & $\begin{array}{l}\text { Algunos estudiantes esperan a sus compañeros/as a la salida del } \\
\text { colegio para seguir golpeándolos o intimidándolos. }\end{array}$ & $\square$ & $\square$ & $\square$ & $\square$ \\
\hline 13. & Algunos estudiantes pelean en las cercanías del colegio. & $\square$ & $\square$ & $\square$ & $\square$ \\
\hline 14. & $\begin{array}{l}\text { Ciertos estudiantes amenazan con golpes a otros compañeros/as } \\
\text { para causarles miedo u obligarles a hacer cosas. }\end{array}$ & $\square$ & $\square$ & $\square$ & $\square$ \\
\hline
\end{tabular}




\begin{tabular}{|c|c|c|c|c|c|}
\hline 15. & $\begin{array}{l}\text { Algunos estudiantes dan patadas, empujones o combos a sus } \\
\text { compañeros/as bromeando o para dejarles en vergüenza. }\end{array}$ & $\square$ & $\square$ & $\square$ & $\square$ \\
\hline 16. & $\begin{array}{l}\text { Hay estudiantes que avergüenzan a otros compañeros/as con } \\
\text { bromas pesadas haciéndoles quedar en ridículo. }\end{array}$ & $\square$ & $\square$ & $\square$ & $\square$ \\
\hline 17. & $\begin{array}{l}\text { En el colegio algunos compañeros hacen bromas como encerrar a } \\
\text { otros o esconder sus cosas para burlarse. }\end{array}$ & $\square$ & $\square$ & $\square$ & $\square$ \\
\hline 18. & $\begin{array}{l}\text { En el colegio algunos compañeros/as rompen o roban las cosas de } \\
\text { otros para burlarse o intimidarlos. }\end{array}$ & $\square$ & $\square$ & $\square$ & $\square$ \\
\hline 19. & $\begin{array}{l}\text { En el colegio hay estudiantes que hacen dibujos en papeles o } \\
\text { murallas para ofender a otros compañeros/as. }\end{array}$ & $\square$ & $\square$ & $\square$ & $\square$ \\
\hline 20. & $\begin{array}{l}\text { Ciertos estudiantes se comportan desafiantes o prepotentes con los } \\
\text { profesores. }\end{array}$ & $\square$ & $\square$ & $\square$ & $\square$ \\
\hline 21. & $\begin{array}{l}\text { Algunos estudiantes rompen o deterioran a propósito muebles o } \\
\text { materiales que son del colegio. }\end{array}$ & $\square$ & $\square$ & $\square$ & $\square$ \\
\hline 22. & $\begin{array}{l}\text { En el colegio hay estudiantes que se sienten solos en las clases, } \\
\text { ignorados o rechazados por sus compañeros/as. }\end{array}$ & $\square$ & $\square$ & $\square$ & $\square$ \\
\hline 23. & $\begin{array}{l}\text { Algunos estudiantes son discriminados o burlados por sus } \\
\text { compañeros/as por tener buenas notas, y ser considerados 'nerds' } \\
\text { o 'mateos'. }\end{array}$ & $\square$ & $\square$ & $\square$ & $\square$ \\
\hline 24. & Algunos estudiantes son discriminados por sus bajas notas. & $\square$ & $\square$ & $\square$ & $\square$ \\
\hline 25. & $\begin{array}{l}\text { Hay estudiantes que no dejan entrar en su grupo a otros } \\
\text { compañeros/as haciéndoles sentir que son inferiores. }\end{array}$ & $\square$ & $\square$ & $\square$ & $\square$ \\
\hline 26. & $\begin{array}{l}\text { En mi curso existe un grupo que se cree superior o 'popular' y que } \\
\text { generalmente excluye o discrimina al resto. }\end{array}$ & $\square$ & $\square$ & $\square$ & $\square$ \\
\hline 27. & $\begin{array}{l}\text { Hay estudiantes que son discriminados o excluidos por su físico, o } \\
\text { porque son lentos o algo les cuesta más que los demás compañeros/as. }\end{array}$ & $\square$ & $\square$ & $\square$ & $\square$ \\
\hline 28. & $\begin{array}{l}\text { Algunos estudiantes son discriminados o excluidos por sus } \\
\text { compañeros/as por su personalidad o su forma de ser. }\end{array}$ & $\square$ & $\square$ & $\square$ & $\square$ \\
\hline 29. & $\begin{array}{l}\text { Hay estudiantes que se quedan solos en los recreos o en las } \\
\text { actividades extraescolares porque son ignorados o rechazados por } \\
\text { sus compañeros/as. }\end{array}$ & $\square$ & $\square$ & $\square$ & $\square$ \\
\hline 30. & $\begin{array}{l}\text { Hay estudiantes que son discriminados o excluidos por sus } \\
\text { compañeros/as por ser indígenas o extranjeros. }\end{array}$ & $\square$ & $\square$ & $\square$ & $\square$ \\
\hline 31. & $\begin{array}{l}\text { Hay compañeros/as que avergüenzan o ridiculizan a otros para } \\
\text { dañar su imagen, excluirlos o dejarles fuera de los encuentros } \\
\text { sociales del curso. }\end{array}$ & $\square$ & $\square$ & $\square$ & $\square$ \\
\hline 32. & $\begin{array}{l}\text { En mi colegio algunos estudiantes ofenden, insultan o amenazan a } \\
\text { otros a través de mensajes de texto por WhatsApp, Line, correo } \\
\text { electrónico u otros. }\end{array}$ & $\square$ & $\square$ & $\square$ & $\square$ \\
\hline
\end{tabular}




\begin{tabular}{|c|c|c|c|c|c|}
\hline 33. & $\begin{array}{l}\text { En mi colegio hay estudiantes que ofenden, insultan o amenazan a } \\
\text { otros por medio de redes sociales como Facebook, Tuenti, ask.fm } \\
\text { u otros. }\end{array}$ & $\square$ & $\square$ & $\square$ & $\square$ \\
\hline 34. & $\begin{array}{l}\text { En mi colegio algunos estudiantes publican o comparten fotos o } \\
\text { vídeos ofensivos de compañeros/as para burlarse. }\end{array}$ & $\square$ & $\square$ & $\square$ & $\square$ \\
\hline 35. & $\begin{array}{l}\text { Algunos estudiantes hacen comentarios, o suben fotos o videos de } \\
\text { compañeros/as para avergonzarlos o dañar su imagen. }\end{array}$ & $\square$ & $\square$ & $\square$ & $\square$ \\
\hline 36. & $\begin{array}{l}\text { Hay estudiantes que graban videos o hacen fotos a compañeros/as } \\
\text { para amenazarles o chantajearles. }\end{array}$ & $\square$ & $\square$ & $\square$ & $\square$ \\
\hline 37. & $\begin{array}{l}\text { En el colegio algunos estudiantes publican o comparten fotos o } \\
\text { vídeos ofensivos de profesores. }\end{array}$ & $\square$ & $\square$ & $\square$ & $\square$ \\
\hline 38. & $\begin{array}{l}\text { Ciertos estudiantes ofenden, insultan o amenazan a los profesores, } \\
\text { por medio de redes sociales como Facebook, Tuenti, ask.fm, u } \\
\text { otros. }\end{array}$ & $\square$ & $\square$ & $\square$ & $\square$ \\
\hline 39. & $\begin{array}{l}\text { Algunos estudiantes crean cuentas o perfiles en redes sociales con } \\
\text { nombres falsos para difundir comentarios que avergüenzan o } \\
\text { intimidan a otros. }\end{array}$ & $\square$ & $\square$ & $\square$ & $\square$ \\
\hline 40. & $\begin{array}{l}\text { Hay estudiantes que se apropian del Facebook o Correo de otros } \\
\text { compañeros, para ver contenido privado, burlarse, amenazar o } \\
\text { agredir. }\end{array}$ & $\square$ & $\square$ & $\square$ & $\square$ \\
\hline 41. & $\begin{array}{l}\text { En el colegio hay profesores que le tienen mala barra a algunos } \\
\text { compañeros/as. }\end{array}$ & $\square$ & $\square$ & $\square$ & $\square$ \\
\hline 42. & $\begin{array}{l}\text { En el colegio algunos profesores se burlan o ridiculizan a algunos } \\
\text { estudiantes. }\end{array}$ & $\square$ & $\square$ & $\square$ & $\square$ \\
\hline 43. & $\begin{array}{l}\text { En el colegio hay algunos profesores que hacen sus clases sin } \\
\text { escuchar a la mayoría de los estudiantes o ignorándolos. }\end{array}$ & $\square$ & $\square$ & $\square$ & $\square$ \\
\hline 44. & $\begin{array}{l}\text { En el colegio ciertos profesores hacen comentarios que ofenden o } \\
\text { intimidan a algunos estudiantes. }\end{array}$ & $\square$ & $\square$ & $\square$ & $\square$ \\
\hline 45. & $\begin{array}{l}\text { Hay profesores que intencionan malas notas hacia determinados } \\
\text { estudiantes. }\end{array}$ & $\square$ & $\square$ & $\square$ & $\square$ \\
\hline 46. & $\begin{array}{l}\text { En el colegio no nos atrevemos a presentar reclamos cuando un } \\
\text { profesor trata injustamente a un alumno. }\end{array}$ & $\square$ & $\square$ & $\square$ & $\square$ \\
\hline 47. & $\begin{array}{l}\text { En el colegio si tenemos un conflicto con algún profesor, no lo } \\
\text { reflexionamos con él para llegar a un acuerdo. }\end{array}$ & $\square$ & $\square$ & $\square$ & $\square$ \\
\hline 48. & $\begin{array}{l}\text { Algunos profesores llaman la atención con gritos y golpes en la } \\
\text { mesa. }\end{array}$ & $\square$ & $\square$ & $\square$ & $\square$ \\
\hline 49. & $\begin{array}{l}\text { Hay profesores que con sus manos toman fuertemente a los } \\
\text { estudiantes para llamarles la atención o sacarlos de la sala. }\end{array}$ & $\square$ & $\square$ & $\square$ & $\square$ \\
\hline 50. & $\begin{array}{l}\text { En clases los profesores nos ayudan a reflexionar y resolver los } \\
\text { conflictos que existen entre compañeros/as. }\end{array}$ & $\square$ & $\square$ & $\square$ & $\square$ \\
\hline
\end{tabular}




\begin{tabular}{|c|c|c|c|c|c|}
\hline 51. & $\begin{array}{l}\text { En este colegio, reflexionamos junto a los profesores sobre las } \\
\text { consecuencias que tiene el maltrato, la violencia o el abuso. }\end{array}$ & $\square$ & $\square$ & $\square$ & $\square$ \\
\hline 52. & $\begin{array}{l}\text { En el colegio hemos hablado sobre las consecuencias de la } \\
\text { violencia a través de medios sociales. }\end{array}$ & $\square$ & $\square$ & $\square$ & $\square$ \\
\hline 53. & $\begin{array}{l}\text { Los estudiantes reflexionamos junto a los profesores sobre } \\
\text { situaciones que nos pueden llevar a conductas violentas. }\end{array}$ & $\square$ & $\square$ & $\square$ & $\square$ \\
\hline 54. & $\begin{array}{l}\text { Los profesores nos han enseñado a controlar el enojo y evitar la } \\
\text { violencia para resolver pacíficamente a los conflictos. }\end{array}$ & $\square$ & $\square$ & $\square$ & $\square$ \\
\hline 55. & $\begin{array}{l}\text { La escuela hace talleres con los apoderados para prevenir } \\
\text { situaciones de violencia o robo en el colegio. }\end{array}$ & $\square$ & $\square$ & $\square$ & $\square$ \\
\hline 56. & $\begin{array}{l}\text { La escuela hace talleres con los apoderados para hablar de } \\
\text { violencia y maltrato en las redes sociales. }\end{array}$ & $\square$ & $\square$ & $\square$ & $\square$ \\
\hline 57. & $\begin{array}{l}\text { En este colegio los profesores promueven que los estudiantes se } \\
\text { disculpen cuando lastiman a alguien. }\end{array}$ & $\square$ & $\square$ & $\square$ & $\square$ \\
\hline 58. & $\begin{array}{l}\text { Los estudiantes tenemos espacios para hablar sobre los conflictos } \\
\text { que ocurren entre compañeros y logramos llegar a acuerdos para } \\
\text { resolverlos. }\end{array}$ & $\square$ & $\square$ & $\square$ & $\square$ \\
\hline 59. & $\begin{array}{l}\text { En este colegio los estudiantes hemos hecho acciones que nos han } \\
\text { ayudado a disminuir la violencia y mejorar la convivencia en la } \\
\text { sala de clases. }\end{array}$ & $\square$ & $\square$ & $\square$ & $\square$ \\
\hline 60. & $\begin{array}{l}\text { En este colegio se organizan eventos como campañas y talleres } \\
\text { para prevenir situaciones de violencia y acoso entre estudiantes. }\end{array}$ & $\square$ & $\square$ & $\square$ & $\square$ \\
\hline 61. & $\begin{array}{l}\text { Hay espacios en la semana (como las clases de orientación) en que } \\
\text { nos dedicamos como curso a conocernos y aprender a convivir. }\end{array}$ & $\square$ & $\square$ & $\square$ & $\square$ \\
\hline 62. & $\begin{array}{l}\text { En este colegio se aplican sanciones a los estudiantes que } \\
\text { maltratan, discriminan o son violentos con otros }\end{array}$ & $\square$ & $\square$ & $\square$ & $\square$ \\
\hline 63. & $\begin{array}{l}\text { En este colegio se aplican con claridad las sanciones cuando hay } \\
\text { violencia o abuso entre compañeros/as. }\end{array}$ & $\square$ & $\square$ & $\square$ & $\square$ \\
\hline 64. & $\begin{array}{l}\text { En este colegio todos los profesores saben qué hacer para resolver } \\
\text { un conflicto. }\end{array}$ & $\square$ & $\square$ & $\square$ & $\square$ \\
\hline 65. & $\begin{array}{l}\text { En este colegio se difunden las normas que hablan de no insultar } \\
\text { ni golpear a las demás personas. }\end{array}$ & $\square$ & $\square$ & $\square$ & $\square$ \\
\hline 66. & $\begin{array}{l}\text { En este colegio se refuerzan las normas sobre no hacer bromas } \\
\text { pesadas, porque causan daño a los compañeros/as. }\end{array}$ & $\square$ & $\square$ & $\square$ & $\square$ \\
\hline 67. & $\begin{array}{l}\text { En este colegio se enseñan normas que hablan de no robar ni } \\
\text { tomar las cosas de los demás. }\end{array}$ & $\square$ & $\square$ & $\square$ & $\square$ \\
\hline 68. & $\begin{array}{l}\text { En este colegio cuando un estudiante acosa o violenta a otro, los } \\
\text { padres son citados por el inspector o profesor jefe. }\end{array}$ & $\square$ & $\square$ & $\square$ & $\square$ \\
\hline 69. & $\begin{array}{l}\text { El colegio ofrece orientación a los padres de estudiantes que han } \\
\text { sido víctimas de violencia. }\end{array}$ & $\square$ & $\square$ & $\square$ & $\square$ \\
\hline
\end{tabular}




\begin{tabular}{|c|l|l|l|l|l|}
\hline 70. & $\begin{array}{l}\text { En este colegio se hace seguimiento a los estudiantes que han } \\
\text { participado como agresores o víctimas en situaciones de violencia. }\end{array}$ & $\square$ & $\square$ & $\square$ \\
\hline 71. & $\begin{array}{l}\text { Existen personas capacitadas en este colegio, para ayudar a los } \\
\text { estudiantes que presentan problemas de violencia. }\end{array}$ & $\square$ & $\square$ & $\square$ \\
\hline 72. & $\begin{array}{l}\text { Los estudiantes participamos en la elaboración del reglamento de } \\
\text { convivencia escolar, sus normas y sanciones. }\end{array}$ & $\square$ & $\square$ & $\square$ \\
\hline 73. & Analizamos en grupo sobre el por qué de las normas del colegio. & $\square$ & $\square$ & $\square$ & $\square$ \\
\hline 74. & $\begin{array}{l}\text { Los estudiantes ayudamos para que se cumplan las normas } \\
\text { acordadas. }\end{array}$ & $\square$ & $\square$ & $\square$ & $\square$ \\
\hline 75. & $\begin{array}{l}\text { En nuestro colegio o curso nos piden organizarnos para defender- } \\
\text { nos y que nunca ocurra bullying. }\end{array}$ & $\square$ & $\square$ & $\square$ & $\square$ \\
\hline 76. & $\begin{array}{l}\text { Cuando sabemos de algo grave que está pasando en el colegio, lo } \\
\text { informamos con confianza al director/a, inspectores o profesores. }\end{array}$ & $\square$ & $\square$ & $\square$ & $\square$ \\
\hline 77. & $\begin{array}{l}\text { Si un estudiante está sufriendo maltrato de parte de otros, puede } \\
\text { pedir ayuda a un adulto del colegio. }\end{array}$ & $\square$ & $\square$ & $\square$ & $\square$ \\
\hline 78. & $\begin{array}{l}\text { Los estudiantes nos sentimos escuchados por el director/a, } \\
\text { inspectores y profesores. }\end{array}$ & $\square$ & $\square$ & $\square$ & $\square$ \\
\hline 79. & $\begin{array}{l}\text { Entre nosotros nos apoyamos cuando vemos que alguien sufre } \\
\text { violencia. }\end{array}$ & $\square$ & $\square$ & $\square$ & $\square$ \\
\hline
\end{tabular}

¡MUCHAS GRACIAS! 
